\title{
How do the experiences and beliefs of adults and children with heterozygous familial hypercholesterolaemia influence their adherence to treatment? A systematic review of qualitative evidence protocol
}

\author{
Fiona J. Kinnear ${ }^{1,2^{*}}$ (D), Rachel Perry ${ }^{1,2}$, Aidan Searle ${ }^{1,2}$, Julian P. Hamilton-Shield ${ }^{1,2}$ and Fiona E. Lithander ${ }^{1,2}$
}

\begin{abstract}
Background: Heterozygous familial hypercholesterolaemia $(\mathrm{FH})$ is a genetic disorder characterised by elevated levels of low density lipoprotein (LDL) cholesterol from birth, estimated to affect 1 in 250 of the UK population. Left untreated, FH substantially increases an individual's risk of premature coronary heart disease (CHD) and associated mortality. This risk can be minimised with timely diagnosis and successful treatment with medication and lifestyle changes, as advocated in national and international guidelines. Despite these recommendations, the limited research available suggests adherence to treatment may be sub-optimal. This review will identify and synthesise the available qualitative research regarding the experiences and beliefs of adults and children with $\mathrm{FH}$ in relation to their condition and its treatment, and the influence of these upon treatment adherence.
\end{abstract}

Methods: The following electronic databases will be searched from their inception: Cochrane library, MEDLINE, Embase, PsycINFO (via OVID) and CINAHL. Studies available in English and reporting primary qualitative data will be included. Database searching will be supplemented with searches in relevant specialist websites. The references of identified papers will also be hand searched. Two reviewers will independently screen titles and abstracts of identified studies, with full texts of potentially relevant papers retrieved for review against pre-defined inclusion and exclusion criteria. The Critical Appraisal Skills Programme (CASP) Qualitative Research checklist will be used to assess quality of the included studies, and the results will be taken into consideration when reporting the findings. A data extraction tool will be created for use in this review to extract study findings relevant to the review questions. A thematic synthesis approach will be taken to analyse the results.

Discussion: Adherence to treatment recommendations is crucial for the successful management of $\mathrm{FH}$ and subsequent decrease in risk of CHD later in life. Common identified themes could provide an understanding of the beliefs and experiences which influence adherence to treatment recommendations and provide an insight into perceived barriers and facilitators. The findings are intended to be used in the development of future interventions or guidelines regarding treatment of children and adults with $\mathrm{FH}$.

Systematic review registration: PROSPERO registration number: CRD42018085946

Keywords: Familial hypercholesterolaemia, Children, Adolescents, Diet, Physical activity, Cholesterol, Systematic review, Qualitative research, Treatment adherence, Cardiovascular heart disease

\footnotetext{
*Correspondence: Fiona.kinnear@bristol.ac.uk

${ }^{1} \mathrm{NIHR}$ Bristol Biomedical Research Centre (Nutrition Theme), University

Hospitals Bristol NHS Foundation Trust, Bristol, UK

${ }^{2}$ University of Bristol, Bristol, UK
}

(c) The Author(s). 2018 Open Access This article is distributed under the terms of the Creative Commons Attribution 4.0 International License (http://creativecommons.org/licenses/by/4.0/), which permits unrestricted use, distribution, and reproduction in any medium, provided you give appropriate credit to the original author(s) and the source, provide a link to the Creative Commons license, and indicate if changes were made. The Creative Commons Public Domain Dedication waiver (http://creativecommons.org/publicdomain/zero/1.0/) applies to the data made available in this article, unless otherwise stated. 


\section{Background}

Familial hypercholesterolaemia $(\mathrm{FH})$ is an autosomal dominant hereditary disorder, characterised by markedly elevated levels of low-density lipoprotein (LDL) cholesterol from birth [1]. This review will focus on heterozygous $\mathrm{FH}$ (henceforth to be known as FH) which has been estimated to affect 1 in 250 of the UK population [2] and substantially increases risk of premature coronary heart disease (CHD) and associated mortality [3, 4]. However, this risk can be reduced with appropriate lifelong treatment [5]. UK and international guidance recommends that individuals with $\mathrm{FH}$ should commence life-long lipid-lowering drug therapy from 10 years of age, alongside the adoption of lifestyle advice which aims to encourage maintenance of a healthy weight, smoking avoidance, participation in regular physical activity (PA), limited alcohol consumption and adherence to a healthy, balanced diet $[6,7]$.

There are very little data regarding medication and lifestyle advice provision and adherence in the $\mathrm{FH}$ population [8]. From the available evidence, it appears that healthcare professionals are following the guidelines with the majority of adults [9], but treatment provided to children is inconsistent $[4,10]$. While adherence to medication in adults appears to be high [11-13], a Dutch questionnaire survey found that only $49 \%$ of adults with $\mathrm{FH}$ adhere to dietary and PA recommendations [11]. Similarly, the adoption of lifestyle recommendations has been reported to be low in children [10].

Qualitative research can provide an insight into an individual's understanding, perceptions and beliefs of their condition and its management. This can enable the development of an understanding of how and why individuals' display certain behaviours and an awareness of factors influencing their decisions to adhere to treatment [14]. Illness perceptions and beliefs about treatment have been found to influence treatment compliance in adults with chronic conditions such as asthma $[15,16]$, epilepsy [17] and hypertension [18]. Specifically, necessity and concern beliefs have been found to be modifiers of treatment adherence across a range of health conditions [19]. Similar findings have been found for children and adolescents with asthma [20], cystic fibrosis [21] and type 1 diabetes [22]. Within the FH population, adherence to treatment has been found to be influenced by several factors including knowledge of $\mathrm{FH}$ [23], perception of risk [24], beliefs about influence of treatment upon long-term health [23] and personal experiences such as family history of cardiovascular disease [25]. Furthermore, barriers to adhering to treatment have been highlighted including a lack of motivation [23], social acceptance [24] and concerns regarding medication [26]. From the limited research conducted in young adults with $\mathrm{FH}$, experiences of family history of disease has been found to influence decisions regarding treatment [25] and lack of credible information, motivation and symptoms highlighted as barriers to adhering to treatment [25, 27].

Results from these individual studies provide useful insights but are restricted by their small sample sizes and are subject to bias resulting from the researcher's interpretation of the participants' views, and the quotes they select to present in the findings. By conducting a systematic review of qualitative studies, a wider range of patient perceptions and experiences can be examined which allows for common themes to be identified and explored in more depth [28]. Furthermore, it could provide awareness of common enablers and barriers to treatment adherence within the patient population group. These findings would be useful in the development of future interventions or recommendations for the $\mathrm{FH}$ population. To date, one systematic review of qualitative research in the FH population has been carried out [29]; however, it aimed to answer questions regarding the influence of screening approaches on diagnostic rates. Thus far, no synthesis of qualitative data regarding the experiences and beliefs of individuals with $\mathrm{FH}$ in relation to their condition, its treatment and the influence of these upon treatment adherence has been carried out.

\section{Research objective}

This systematic review aims to identify and synthesise the available qualitative evidence regarding the experiences and beliefs of adults and children with $\mathrm{FH}$ in relation to their condition, its treatment and the influence of these upon treatment adherence. This review also seeks to identify any perceived enablers and barriers to treatment adherence. The findings are intended to be used in the development of future interventions or guidelines targeting medication and lifestyle behaviours in children and adults with $\mathrm{FH}$.

\section{Research questions}

In adults and children with $\mathrm{FH}$ :

- What are the experiences and beliefs of individuals' in relation to their condition, its associated morbidity and mortality risk and treatment?

- How do these experiences and beliefs influence individuals' adherence to pharmacological and lifestyle treatment recommendations?

- Are there any enablers and/or barriers to adhere to pharmacological and lifestyle treatment recommendations?

- Do the findings differ between adults and children?

\section{Methods}

Where appropriate for qualitative reviews, the reporting of this protocol is in accordance with the 'Preferred Reporting Items for Systematic Review and Meta-Analysis 
Protocols (PRISMA-P) 2015 checklist' [30]. A completed checklist for this review protocol is available in Additional file 1. This protocol will also be reported in accordance with the enhancing transparency in reporting the synthesis of qualitative research (ENTREQ) statement [31].

\section{Eligibility criteria}

Papers reporting studies that meet the following criteria will be included in the analysis.

\section{Participants}

The participants include individuals aged $\geq 10$ years with clinically diagnosed $\mathrm{FH}$. In this review, a genetic diagnosis of $\mathrm{FH}$ is not required for inclusion if the study clearly states that the study population has all received a clinical diagnosis. The age of 10 years was used as a cutoff as this is age that children with FH are currently advised to be considered for treatment with statins and to attend subsequent follow-up appointments in a FH specialist clinic in the UK [7]. Individuals with a history of cardiovascular heart disease will be included. Individuals with a diagnosis of homozygous $\mathrm{FH}$ will be excluded.

\section{Phenomena of interest}

This review will consider any studies that investigate the experiences and beliefs of individuals with clinically diagnosed FH in relation to their condition, its associated morbidity and mortality risk and recommended pharmacological and lifestyle change treatment. Studies focusing on specific elements of $\mathrm{FH}$ which are unrelated to the research objectives of this report, such as attitudes towards genetic testing, will be included if the study also includes findings relevant to this review which can be independently extracted. Studies in which the focus is upon the perceptions and experiences of the family members, for example, parents of children and adolescents with $\mathrm{FH}$, will also be included.

\section{Type of studies}

This review aims to explore the experiences and beliefs of individuals which are most appropriately done through analysis of qualitative data, and as such, only qualitative studies that report primary data will be included. For the purpose of this review, qualitative research refers to studies in which widely recognised qualitative methods of data collection and data analysis have been used. This includes, but is not limited to, data collection methods such as focus groups and interviews, and data analyses methods including narrative analysis and grounded theory [32]. Only studies in which the full text is available in English will be eligible for inclusion. This decision was taken due to the potential problems in interpreting and translating qualitative data in another language by the research team and lack of resource to carry this out. Mixed method studies, in which both quantitative and qualitative methods were used, will be included if it is possible to extract only the qualitative data. Quantitative studies, commentaries or reviews on the subject will be excluded. Studies which exclusively collected information through self-reported questionnaires or researcher administered surveys will be excluded as this does not allow for an in-depth understanding of individuals' beliefs.

\section{Intervention/exposure}

In this review, treatment is defined as any action taken by individuals with $\mathrm{FH}$ in an attempt to manage their condition as a result of their own knowledge of the condition, advice given to them by a healthcare professional or from other family members. This includes, but is not limited to, behaviours relating to medication, weight management, smoking, diet and physical activity. Variations in the length of time since diagnosis and the mode of delivery, intensity and content of treatment advice which individuals will have received are expected; however, no exclusions will be made based on this criteria.

\section{Setting}

Studies will be included regardless of the country in which they were conducted and the setting in which the qualitative data were collected from individuals.

\section{Search strategy}

The search will be systematic, pre-planned and comprehensive in order to find all available studies, as recommended for thematic synthesis $[31,33]$. The search strategy will be developed by two reviewers (FK, RP) with reference to the available guidance and recommendations [31, 34, 35], as well as consultation with an information specialist with previous experience in designing qualitative search strategies. It will be carried out by one reviewer (RP).

\section{Electronic searches}

Literature searches will be undertaken in the following databases for published articles: MEDLINE, Embase, PsycINFO (via OVID), Cochrane library and CINAHL. All will be searched from the inception of the database to present. Search terms relating to the population will be combined with a validated search filter [36] containing terms relating to qualitative methods and methodology. The search will use a combination of subject headings (e.g. MeSH) and text words. A draft of the search strategy intended for use in MEDLINE is available in Additional file 2. The same search strategy will be used in the other listed databases, with appropriate subject headings and database-specific modifications to syntax and qualitative search filter applied. 


\section{Searching other resources}

The reference lists of all included studies will be hand searched for additional studies. As qualitative research is often found in grey literature such as conference abstracts and theses [31], the OpenGrey database will be searched. Relevant specialist websites, including charities and government health departments with a focus in lipid, cardiac or genetic disorders will also be searched, e.g. American Heart Association, the international FH Foundation, the British Heart Foundation and the Simon Broome Register. Authors of any identified abstracts will be contacted to establish if full text is available. Experts in the area will be contacted to enquire about the existence of any unpublished work.

\section{Data management}

The search results will be managed using Endnote reference management software. The results from the database searches will be imported into an Endnote library, with duplicates removed during the screening of the data.

\section{Selection of studies}

Two reviewers (FK, RP) will independently screen the titles and abstracts of all retrieved records for inclusion. Full-text copies of potentially relevant studies will be retrieved for in-depth review against the inclusion criteria. The full-text articles will be screened independently by two reviewers (FK, JHS). Explanations for exclusion will be recorded at the full-text stage. A Preferred Reporting Items for Systematic Review and Meta-Analysis (PRISMA) flow chart will be produced to illustrate the different stages of study selection. Full-text articles which are judged to meet inclusion criteria will be taken forward to appraisal and data extraction stages.

\section{Data extraction}

Firstly, the contextual and methodological information of the included studies will be extracted into a study table designed for this review, as recommended [34]. Information on the following will be extracted:

- Publication information: author, year of publication, country of study

- Sample characteristics: number of participants, demographics, FH diagnostic criteria

- Study design: methodological approach, conceptual bases underlying study, analysis method, setting

- Study objectives or aims

Secondly, in line with Thomas and Harden's approach [33], study findings (data) will be extracted using a tool designed specifically for this review. All text and quotations reported in the 'results/findings,' 'discussion' and 'conclusion' sections of papers, as well as in any supplemental files provided by the authors, will be considered for extraction. This inclusive approach to data extraction has been taken to minimise risk of missing findings which may be of potential value to the synthesis, as it is recognised that authors may report useful data out with the 'findings' section [34]. Any data judged to be relevant to the research questions of this review will be extracted. This data will take the form of first-order constructs (participants' quotes and author summaries of quotes) and second-order constructs (author's interpretations).

Both stages of data extraction will be carried out independently by two reviewers (FK, AS), with any disagreement resolved by discussion until consensus is reached, with a third reviewer (FL) consulted if required. The data extraction tool will be piloted on the first five studies, after which its suitability for use will be discussed and any required amendments made before continuation of data extraction on the remaining studies. Microsoft Excel or NVivo software will be used to manage the extracted data.

\section{Critical appraisal of study quality}

The Critical Appraisal Skills Programme (CASP) Qualitative Research Checklist [37] will be used to assess the methodological validity of included studies. This will be carried out by two reviewers (FK, AS), with any disagreements discussed with a third reviewer (RP or FL). This tool was chosen as it can be applied to qualitative research of various designs, is frequently used in the literature [31] and is a recommended tool [34].

To avoid the possibility of excluding potentially valuable insights that can be generated from qualitative studies assessed to be inadequately designed or reported [28], the results of the appraisal will not be used to exclude any study. In their worked example of thematic synthesis methodology, Thomas and Harden [33] chose not to exclude studies as there is no widely accepted and empirically tested method for excluding studies based on their quality. However, it is recommended that the quality of included studies should be critically analysed in the subsequent data synthesis and be considered when drawing conclusions in systematic reviews of qualitative research [31]. Therefore, the appraisal findings will be presented alongside the findings of the review. Furthermore, after synthesis, the relative contributions of studies to the derived analytic themes and any recommendations which are made based on these will be explored in relation to their evaluated quality.

\section{Data synthesis}

There are many recognised methods for qualitative synthesis, with no gold standard method recommended for use [38]. Instead, it is advised that the choice of method should be informed by the type and scope of review question and the content of the available literature [34]. 
Thematic synthesis is a method developed by Thomas and Harden [33] to answer questions addressing the suitability and acceptability of health promotion and public health interventions. The benefit of including the experiences and perceptions of the individuals being targeted in the interventions when assessing the effectiveness of such interventions was recognised, and thematic synthesis was developed to analyse and understand these qualitative data. The comparable research aims of this review, and its recommendation for use in qualitative systematic reviews [34], led to the decision to choose thematic synthesis.

Thematic synthesis is comprised of three main stagesline by line coding, development of descriptive themes and generation of new analytical themes from consideration of all the available data [33]. Line by line analysis will be carried out on the extracted data, with each first or second order construct identified being assigned codes. These codes will be inductively created in response to the findings uncovered. This will be carried out independently by two reviewers (FK, AS) with a third reviewer (FL) consulted to resolve any disparities. The two reviewers (FK, AS) will then work in collaboration to develop initial descriptive themes and categories based upon the raw data that closely reflect the aggregative findings of the included studies. Lastly, interpretative analytical themes based upon these aggregative findings will be generated where possible by the two reviewers. This stage seeks to go beyond the original findings of the studies to generate additional understanding and aims to produce recommendations for future interventions.

\section{Discussion}

Adherence to treatment recommendations is crucial for the successful management of $\mathrm{FH}$ and subsequent reduction in risk of CHD later in life. This review will summarise the available qualitative evidence regarding the experiences and beliefs of adults and children with $\mathrm{FH}$ in relation to their condition and its treatment. Common identified themes could provide a greater understanding of the beliefs and experiences which influence the extent to which individuals follow current treatment recommendations and provide an insight into perceived barriers and facilitators to adherence. The findings are intended to be used in the development of future interventions or guidelines targeting medication and lifestyle behaviours in children and adults with FH.

\section{Additional files}

Additional file 1: PRISMA-P (Preferred Reporting Terms for Systematic Review and Meta-analysis Protocol(s) 2015 checklist: recommended items to address in a systematic review protocol. PRISMA (Preferred Reporting Terms for Systematic Review and Meta-Analysis) is an evidence-based minimum set of terms for reporting in systematic reviews and meta-analyses. (DOCX $29 \mathrm{~kb}$ ) Additional file 2: Draft MEDLINE search strategy. (DOCX $14 \mathrm{~kb}$ )

\section{Abbreviations}

CASP: Critical Appraisal Skills Programme; CHD: Cardiovascular heart disease; ENTREQ: Enhancing transparency in reporting the synthesis of qualitative research; FH: Familial hypercholesterolaemia; LDL: Low-density lipoprotein; MeSH: Medical Subject Headings; PA: Physical activity; PRISMA: Preferred Reporting Items for Systematic review and Meta-Analysis; PRISMA-P: Preferred Reporting Items for Systematic review and Meta-Analysis Protocols

\section{Acknowledgements}

The authors would like to thank Catherine Borwick (University of Bristol) for her contribution to the search strategy and Dr. Alison Gregory (University of Bristol) and Dr. Alyson Huntley (University of Bristol) for their advice and guidance on developing protocols for systematic reviews of qualitative research.

\section{Funding}

This systematic review is funded by the National Institute for Health Research (NIHR) Bristol Biomedical Research Centre (Nutrition theme) at University Hospitals Bristol NHS Foundation Trust and the University of Bristol. The views expressed in this publication are those of the authors and not necessarily those of the NHS, the NIHR or the Department of Health.

\section{Authors' contributions}

FK, FL, AS and RP conceptualised and designed the review protocol. RS and FK developed the search strategy. FK prepared the manuscript and was supervised throughout by JHS and FL. All authors revised the manuscript and approved the final version.

\section{Ethics approval and consent to participate}

Not applicable.

\section{Consent for publication}

Not applicable.

\section{Competing interests}

The authors declare that they have no competing interests.

\section{Publisher's Note}

Springer Nature remains neutral with regard to jurisdictional claims in published maps and institutional affiliations.

Received: 25 January 2018 Accepted: 2 August 2018

Published online: 16 August 2018

\section{References}

1. Goldstein $\mathrm{L}$, Hobbs HH, Brown MS. Familial hypercholesterolemia. In: Sciver CRA, Sly WS, Valle D, editors. The metabolic and molecular bases of inherited disease. 8th ed. New York: McGraw-Hill; 2001. p. 2863-913.

2. Akioyamen LE, Genest J, Shan SD, Reel RL, Albaum JM, Chu A, et al. Estimating the prevalence of heterozygous familial hypercholesterolaemia: a systematic review and meta-analysis. BMJ Open. 2017; https://doi.org/10. 1136/bmjopen-2017-016461.

3. Wong B, Kruse G, Kutikova L, Ray KK, Mata P, Bruckert E. Cardiovascular disease risk associated with familial hypercholesterolemia: a systematic review of the literature. Clin Ther. 2016:38:1696-709.

4. Ramaswami U, Cooper J, Humphries SE, on behalf of the FH Paediatric Register Steering Group. The UK paediatric familial hypercholesterolaemia register: preliminary data. Arch Dis Child. 2017;102:255-60.

5. Hovingh GK, Davidson MH, Kastelein JJP, O'Connor AM. Diagnosis and treatment of familial hypercholesterolaemia. Eur Heart J. 2013;34:962-71.

6. Watts GF, Gidding S, Wierzbicki AS, Toth PP, Alonso R, Brown WV, et al. Integrated guidance on the care of familial hypercholesterolaemia from the International FH Foundation. Int J Cardiol. 2014;171:309-25.

7. National Institute for Health and Clinical Excellence. Familial hypercholesterolemia: identification and management. (CG71). 2008. http:// nice.org.uk/guidance/cg71. Accessed 20 Jan 2018.

8. Nordestgaard BG, Chapman MJ, Humphries SE, Ginsberg HN, Masana L, Descamps OS, et al. Familial hypercholesterolaemia is underdiagnosed and undertreated in the general population: guidance for clinicians to prevent coronary heart disease: consensus statement of the European Atherosclerosis Society. Eur Heart J. 2013;34:3478-90. 
9. Hadfield SG, Horara S, Starr BJ, Yazdgerdi S, Bhatnagar D, Cramb R, et al. Are patients with familial hypercholesterolaemia well managed in lipid clinics? An audit of eleven clinics from the Department of Health Familial Hypercholesterolaemia Cascade Testing project. Ann Clin Biochem. 2008;45:199-205.

10. Avis HJ, Kusters DM, Vissers MN, Huijgen $R$, Janssen $T H$, Wiegman $A$, et al. Follow-up of children diagnosed with familial hypercholesterolemia in a national genetic screening program. J Pediatr. 2012;161:99-103.

11. Claassen L, Henneman L, Kindt I, Marteau TM, Timmermans DRM. Perceived risk and representations of cardiovascular disease and preventive behaviour in people diagnosed with familial hypercholesterolemia: a cross-sectional questionnaire study. J Health Psychol. 2010;15:33-43.

12. Langslet G, Bogsrud MP, Halvorsen I, Fjeldstad H, Retterstøl K, Veierød MB, et al. Long-term follow-up of young adults with familial hypercholesterolemia after participation in clinical trials during childhood. J Clin Lipidol. 2015;9:778-85.

13. Huijgen R, Kindt I, Verhoeven SB, Sijbrands EJ, Vissers MN, Kastelein JJ, et al. Two years after molecular diagnosis of familial hypercholesterolemia: majority on cholesterol-lowering treatment but a minority reaches treatment goal. PLoS One. 2010; https:/doi.org/10.1371/journal.pone.0009220.

14. Sutton J, Austin Z. Qualitative research: data collection, analysis, and management. Can J Hosp Pharm. 2015;68:226-31.

15. Horne R, Weinman J. Self-regulation and self-management in asthma: exploring the role of illness perceptions and treatment beliefs in explaining non-adherence to preventer medication. Psychol Health. 2002;17:17-32.

16. Miles C, Arden-Close E, Thomas M, Bruton A, Yardley L, Hankins M, et al. Barriers and facilitators of effective self-management in asthma: systematic review and thematic synthesis of patient and healthcare professional views. NPJ Prim Care Respir Med. 2017;27:57.

17. Chapman SC, Horne R, Chater A, Hukins D, Smithson WH. Patients' perspectives on antiepileptic medication: relationships between beliefs about medicines and adherence among patients with epilepsy in UK primary care. Epilepsy Behav. 2014;31:312-20.

18. Ross S, Walker A, MacLeod MJ. Patient compliance in hypertension: role of illness perceptions and treatment beliefs. J Hum Hypertens. 2004;18:607-13.

19. Foot H, La Caze A, Gujral G, Cottrell N. The necessity-concerns framework predicts adherence to medication in multiple illness conditions: a metaanalysis. Patient Educ Couns. 2016;99:706-17.

20. Holley S, Morris R, Knibb R, Latter S, Liossi C, Mitchell F, et al. Barriers and facilitators to asthma self-management in adolescents: a systematic review of qualitative and quantitative studies. Pediatr Pulmonol. 2017;52:430-42.

21. Bucks RS, Hawkins K, Skinner TC, Horn S, Seddon P, Horne R. Adherence to treatment in adolescents with cystic fibrosis: the role of illness perceptions and treatment beliefs. J Pediatr Psychol. 2009;34:893-902.

22. Griva K, Myers LB, Newman S. Illness perceptions and self efficacy beliefs in adolescents and young adults with insulin dependent diabetes mellitus. Psychol Health. 2000;15:733-50.

23. Hardcastle SJ, Legge E, Laundy CS, Egan SJ, French R, Watts GF, et al. Patients' perceptions and experiences of familial hypercholesterolemia, cascade genetic screening and treatment. Int J Behav Med. 2015;22:92-100.

24. Frich JC, Malterud K, Fugelli P. Experiences of guilt and shame in patients with familial hypercholesterolemia: a qualitative interview study. Patient Educ Couns. 2007;69:108-13.

25. Mackie TI, Tse LL, de Ferranti SD, Ryan HR, Leslie LK. Treatment decision making for adolescents with familial hypercholesterolemia: role of family history and past experiences. J Clin Lipidol. 2015;9:583-93.

26. Mortensen GL, Madsen IB, Kruse C, Bundgaard H. Familial hypercholesterolaemia reduces the quality of life of patients not reaching treatment targets. Dan Med J. 2016;63:A5224.

27. Sliwinski SK, Gooding H, de Ferranti S, Mackie TI, Shah S, Saunders T, et al. Transitioning from pediatric to adult health care with familial hypercholesterolemia: listening to young adult and parent voices. J Clin Lipidol. 2017;11:147-59.

28. Dixon-Woods M, Shaw RL, Agarwal S, Smith JA. The problem of appraising qualitative research. Qual Saf Health Care. 2004;13:223-5.

29. Muir LA, George PM, Whitehead L. Using the experiences of people with familial hypercholesterolaemia to help reduce the risk of cardiovascular disease: a qualitative systematic review. J Adv Nurs. 2012;68:1920-32.

30. Moher D, Shamseer L, Clarke M, Ghersi D, Liberati A, Petticrew M, et al. Preferred reporting items for systematic review and meta-analysis protocols (PRISMA-P) 2015 statement. Syst Rev. 2015;4:1.
31. Tong A, Flemming K, Mclnnes E, Oliver S, Craig J. Enhancing transparency in reporting the synthesis of qualitative research: ENTREQ. BMC Med Res Methodol. 2012;12:181

32. Ritchie J, Lewis J. Qualitative research practice. A guide for social science students and researchers. 1st ed. London: SAGE Publications Ltd; 2003.

33. Thomas J, Harden A. Methods for the thematic synthesis of qualitative research in systematic reviews. BMC Med Res Methodol. 2008:8:45.

34. Noyes J, Booth A, Flemming K, Garside R, Harden A, Lewin S, et al. Cochrane qualitative and implementation methods group guidance paper: methods for assessing methodological limitations, data extraction and synthesis, and confidence in synthesized qualitative findings. J Clin Epidemiol. 2018; https://doi.org/10.1016/j.jclinepi.2017.06.020.

35. Shaw RL, Booth A, Sutton AJ, Miller T, Smith JA, Young B, et al. Finding qualitative research: an evaluation of search strategies. BMC Med Res Methodol. 2004;4:5.

36. McMaster University. Health information research unit. Hedges 2018. https:// hiru.mcmaster.ca/hiru/hiru_hedges_home.aspx. Accessed 31 July 2018.

37. Critical Appraisal Skills Programme. CASP qualitative research checklist. 2017. http://docs.wixstatic.com/ugd/dded87_25658615020e427da194a325e7773d42.pdf. Accessed 20 Jan 2018

38. Barnett-Page E, Thomas J. Methods for the synthesis of qualitative research: a critical review. BMC Med Res Methodol. 2009:9:59.

\section{Ready to submit your research? Choose BMC and benefit from:}

- fast, convenient online submission

- thorough peer review by experienced researchers in your field

- rapid publication on acceptance

- support for research data, including large and complex data types

- gold Open Access which fosters wider collaboration and increased citations

- maximum visibility for your research: over $100 \mathrm{M}$ website views per year

At BMC, research is always in progress.

Learn more biomedcentral.com/submissions 